\title{
Effect of Extraction Process and Surface Treatment on the mechanical properties in Pineapple Leaf Fibre
}

\author{
Azrie Ariffin ${ }^{l}$ and Yusri Yusof ${ }^{2,1}$, \\ ${ }^{1}$ Universiti Tun Hussein Onn Malaysia, (UTHM), Johor, Malaysia
}

\begin{abstract}
Pineapple Leaf Fibre (PALF) is a one of the natural fibre that has high potential in the industry. Natural fibres have become the main alternative source in the modern world industry. The objective of this study is to observe the effect chemical treatment using Sodium Hydroxide $(\mathrm{NaOH})$ solution on the physical and mechanical properties of pineapple leaf fibre. Different concentration of $\mathrm{NaOH}$ solution $(2 \%, 4 \%, 6 \%, 8 \%)$ and different treatment time (1 hour, 3 hour and 5 hour) are used for the experiment. The tensile test was conducted to obtain the mechanical properties such as tensile strength, Yong modulus, (E) and elongation at break. From the results obtained, $\mathrm{NaOH}$ concentration of $6 \%$ and five-hour treatment time that was used for treatment showed the higher physical and mechanical properties values. Furthermore, morphology analysis also shows the surface of the fibre at $6 \% \mathrm{NaOH}$ after five-hour of treatment was in the better condition with good bonding arrangement of the fibre.
\end{abstract}

\section{Introduction}

Natural fibre has begun to pace towards becoming the main alternative source in the modern world industry [1]. Nowadays, pineapple leaf fibre (PALF) is one of the plant fibre source and good natural fibre that has the potential for the alternative source in the industry. (PALF) is used for reinforcement thermoplastic composite for application consumer goods, furniture, textile and civil structures. This type of natural fibre exhibit attractive features such as superior mechanical, physical and thermal properties, thus offer potential uses in a spectrum of applications. Pineapple Leaf Fibre contains only 2.5\%-3.5\% fibre, covered by a hydrophobic waxy layer which remains beneath the waxy layer [2]. In general, fibres are in form of hair-like materials which are consist of continuous filaments or in discrete elongated pieces and most likely like a thread [3]. Recent advances in natural fibre development, genetic engineering, and composites science offer significant opportunities for improved materials from renewable resources with enhanced support for global sustainability [4].

Therefore, in order to increase the mechanical properties of fibre, it may be improved by hydroxyl groups. The process is through the hydrophilization process and consequently enhancing the wetting effect of the resin on the fibres. This combination will be producing a stronger bonded [5]. The research also found that fibre surface roughness and surface area increase after alkaline treatment as shows. It possesses a smaller average diameter of about $44 \mu \mathrm{m}$. Each single fibre can be clearly observed because of removal of some impurities through alkaline treatment. PALF exhibits excellent mechanical properties compared to others. So, in order to improve the mechanical properties of a fibre, a treatment is applied.

\footnotetext{
${ }^{1}$ Corresponding author: azrieyariffin@gmail.com
} 
There are generally consists of coupling agents or compatibilizer agents capable of inducing physical and chemical bonds between the fibre and matrix. For the physical treatments, it changes the structural and surface properties of the fibre and thereby influences the mechanical bonding with the matrix. For the chemical method, they are involving the treatment with stearic acid, coupling agents and also impregnation methods [6].

The alkaline group especially Sodium Hydroxide $(\mathrm{NaOH})$ treatment is an important chemical treatment method used to enhance interfacial adhesion between natural fibre and polymer, as well as the mechanical, physical and thermal properties of the fibre. Alkali treatment is also a good alternative to these chemicals in many cases swell the cell wall of the matrix structure and give better penetration [7]. This treatment also acting to remove hemicellulose, split fibres to fibrils, and lead to a closer packing cellulose chains due to release of internal tension, which in turn increases the mechanical properties of the fibres. For the untreated fibre, it is covered with hemicellulose, lignin, pectin, waxy substances, and natural oils [8].

\section{Methodology}

\subsection{Raw Material Preparation}

Material selection in this study was a natural fibre from pineapple leaves. Pineapple leaf was chosen because of it rich in cellulose, abundantly available and easy to process and handle it. There are two most common types of pineapple planted in Malaysia, which are Josephine and Moris. For this research, Josephine is selected for testing because Josephine shows a better quality in terms of taste, leaf size and even easier to be extracted whilst maintaining a good quality of fibre [9]. Pineapple leaves (Josephine) were extracted by extractor machine called Pineapple Leaf Fibre Machine (PALF M). There were known as wet fibre, dried fibre and usable fibre. Wet fibre state was when the fibre freshly extracted from the leaf which it still contains a lot of moisture. Then, dried fibre state occurs when the fibre was dried at a certain temperature $\left(80^{\circ} \mathrm{C}\right)$. While usable fibre state was when a total of weight of dried fibre divided by the weight of the leaf.

\subsection{Pineapple Leaf Fibre (PALF) Preparation}

For the preparation process, it involved weight, wash, soak, and dry process. After extraction process by using PALF M, PALF was dried under the sun for two days. This experiment required 60 pieces of fibre that have been knotted as shown in Figure 1. PALF was cut approximately twenty centimeters $(20 \mathrm{~cm})$ and the diameter and weight were measured before through the chemical process.

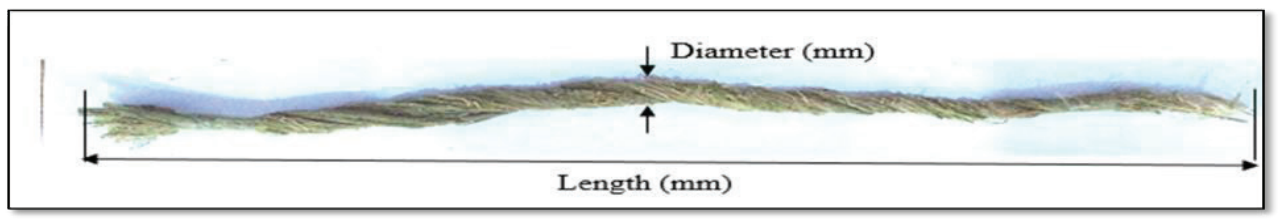

Fig 1. Dimension of PALF 


\subsection{Alkaline Treatment preparation}

The raw material pineapple leaf fibre was undergone alkaline treatment. For this experiment, a Sodium Hydroxide $(\mathrm{NaOH})$ was used as alkaline treatment. The concentration of $\mathrm{NaOH}$ was calculated by using equation (1). Fibres was treated with an alkaline solution to remove natural and artificial impurities which improve the adhesion between the matrix and fibres. Sodium Hydroxide solution also removes the hydrogen bonding in the network structure of the fibre cellulose. The solution also able to increased fibre surface roughness, reduce water absorption and also improving fibre-matrix adhesion.

$$
\text { Concentration } \mathrm{NaOH} \text { solution }=\frac{\text { percentage nesded }}{100} \times \text { total volume of distilled water }
$$

\subsection{Tensile Test of PALF}

The mechanical property of a material was very important in any engineering material. Strength and ability of a product produced depending on the characteristics and mechanical properties of the material. The mechanical properties were calculated by using equations (2)(4). Besides, test results obtained were determined whether an engineering material that can be used in the application or not. For this experiment, mechanical testing methods that were carried out are based on American Standard Testing Methods (ASTM C1557-03). There were two tests performed in the experiment which is a tensile test and morphological analysis with Scanning Electron Microscope (SEM).

Tensile Strength $=\frac{\text { Force }(\text { load })}{\text { crosssectionarea }}$

Young Modulus, $E=\frac{\text { stress }}{\text { strain }}=\frac{F / A}{\Delta L / L}$

Strain,$\varepsilon=$ Elongation Original / elongation

\section{Experimental Result}

\subsection{Chemical Reaction between PALF and Sodium Hydroxide ( $\mathrm{NaOH})$}

Alkaline treatment was an important chemical treatment method used to enhance interfacial adhesion between natural fibre and polymer, as well as the mechanical, physical and thermal properties of the fibre. Figures 2 (a)-(d) show the mechanism of the reaction between the PALF and the $\mathrm{NaOH}$ solution. The reaction shows that the $\mathrm{OH}$ groups are separated from the fibre by the action of the $\mathrm{Na}+2$ ions to produce a new component which is (fibre-NaO) instead of (fibre-OH). $8 \%$ of concentration $\mathrm{NaOH}$ was the most reactive and faster with PALF compared to other concentration because it has a high content of $\mathrm{OH}$ group. In addition, alkali treatment by $\mathrm{NaOH}$ also an alternative to these chemicals in many cases swell the cell wall of the matrix structure and give better penetration [8]. 


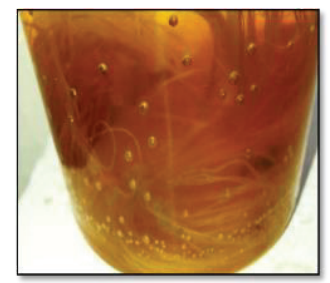

(a): $2 \% \mathrm{NaOH}$

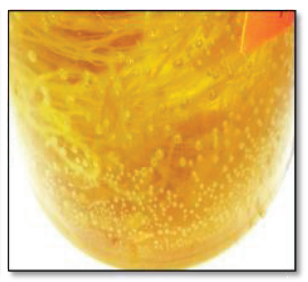

(b): $4 \% \mathrm{NaOH}$

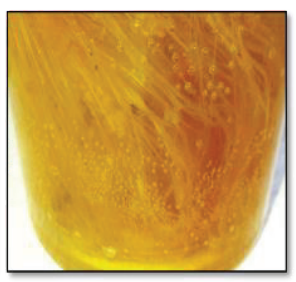

(c): $6 \% \mathrm{NaOH}$

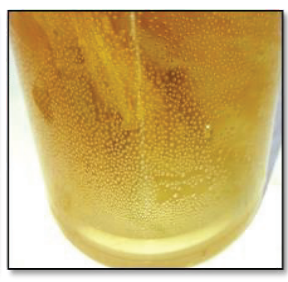

(d): $8 \% \mathrm{NaOH}$

Fig 2. The mechanism of the reaction between the PALF and the $\mathrm{NaOH}$ solution

\subsection{Physical Properties of PALF}

The data shown in Table 1 was the result of weight and diameter of PALF before the chemical treatment. All the PALF was measured and recorded in the table according to the concentration of $\mathrm{NaOH}$ solution. The values of weight and diameter of PALF were different depends on the sizing of pineapple leaves. All the data were taken as a reference to distinguish the result after doing the chemical treatment.

Table 1. Weight and diameter of PALF before an experiment

\begin{tabular}{|c|c|c|}
\hline \multirow{2}{*}{$\begin{array}{c}\text { Concentration } \\
(\%)\end{array}$} & \multicolumn{2}{|c|}{ Weight and Diameter of Pineapple Leaf Fibre (PALF) Before an Experiment (g) } \\
\cline { 2 - 3 } & Weight $(\mathrm{g})$ & Diameter (mm) \\
\hline 2 & 0.8672 & 2.22 \\
\hline 4 & 0.8916 & 2.30 \\
\hline 6 & 0.9851 & 2.46 \\
\hline 8 & 1.0230 & 2.50 \\
\hline
\end{tabular}

Figure 3 shows the higher physical reduction in weight and diameter of PALF after chemical treatment by $\mathrm{NaOH}$ solution for five-hour treatment. From the several conditions that used in the experiment, result for five-hour treatment stated the higher reduction physical properties of PALF. For weight reduction values of PALF, it was directly proportional to the increasing concentration of $\mathrm{NaOH}$ solution. Besides, the reduction value for the diameter of PALF shows a similar trend with a reduction in weight of PALF. Both of data shows that $8 \% \mathrm{NaOH}$ was the better concentration in order to decrease of diameter and weight of PALF. This is because $8 \% \mathrm{NaOH}$ for five-hour treatment give more reaction to the surface of fibre and removal of some hemicelluloses and lignin on the fibre surface. However, the increasing of reduction was affected the tensile strength of PALF.

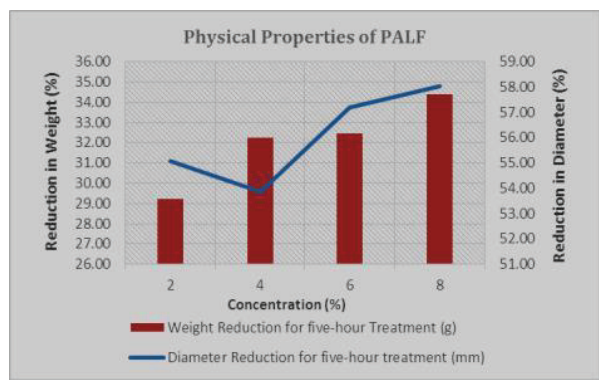

Fig 3. The higher physical properties reduction of PALF after five-hour treatment 


\subsection{Tensile Test of PALF}

Figures 4-5 shows the relationship between the tensile strength, Young modulus, and elongation at break. From the result illustrated in Figure 7, the highest strength of fibre was occurred at $6 \% \mathrm{NaOH}$ for five-hour treatment. At the same graph, elongation at break also gives the likely same trend for the results. The increasing of elongation of fibre was stronger the strength of the fibre. Furthermore, the maximum load required was increased to break the fibre because of strengthen of the fibre itself. Apart of that, Figure 8 shows the Young modulus and tensile strength of PALF. From the analysed, the best result for Young modulus was occur in $2 \%$ concentration of $\mathrm{NaOH}$ after three-hour treatment. The result of the sample was the highest compared to other samples at a different concentration of $\mathrm{NaOH}$ and treatment time.

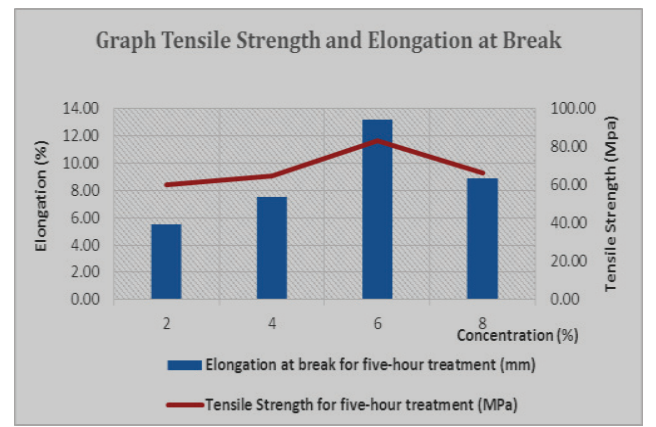

Fig 4. Graph of Tensile Strength and Elongation of PALF for five-hour treatment

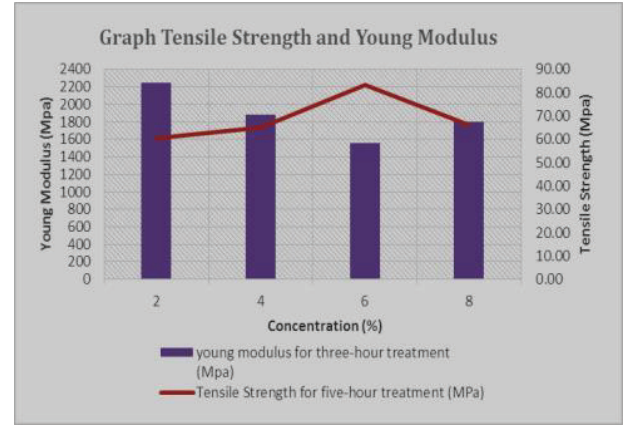

Fig 5. Graph of Tensile Strength and Young modulus, E of PALF for five-hour

For the morphological analysis, the surface structure PALF were different for each test. The results were changed due to the reaction between concentrations of chemical used with the surface of the fibre. According to the result obtained in Figures 6, the best reaction occurs between chemical and fibre at $6 \% \mathrm{NaOH}$ after five-hour treatment. From the morphological analysis, the structure of PALF was well aligned with the matrix and give the good morphology structure. The higher strength and elongation of the fibre are produced from good structure of PALF.

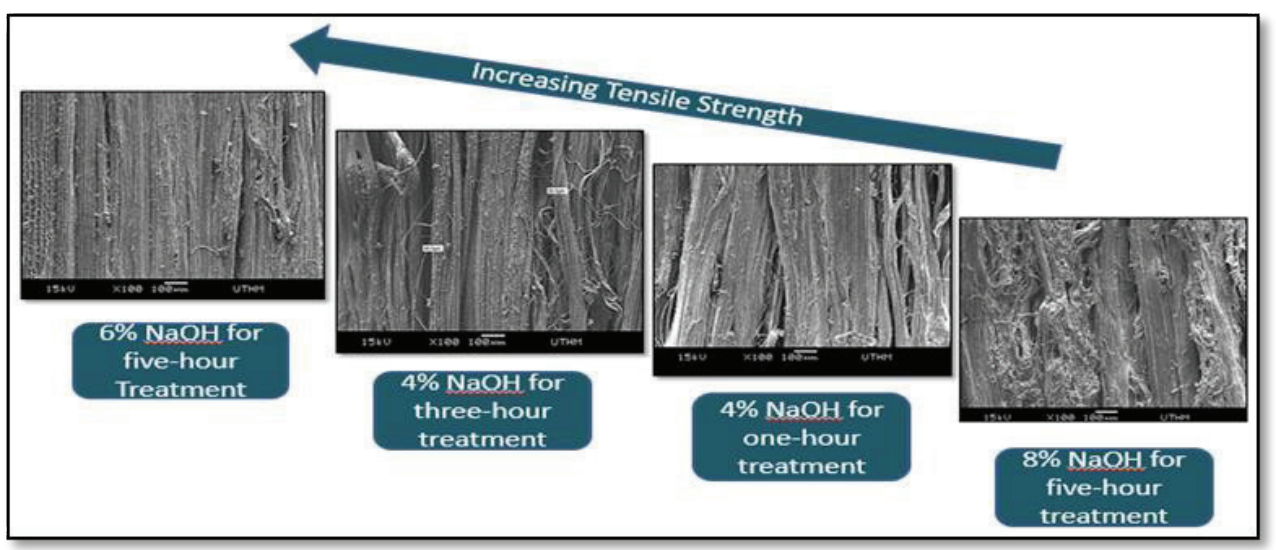

Fig 6. Surface structure of PALF with increasing the concentration of $\mathrm{NaOH}$ 


\section{Conclusion}

From the results, the diameter of PALF was reduced by $38 \%$ from the diameter for untreated PALF before the experiment. The reducing of diameter recorded can be concluded that high concentration $\mathrm{NaOH}$ solution give more reaction to the surface of PALF and more reaction that removed the lignin and wax contained in PALF. Besides, the highest concentration of the chemical solution $(\mathrm{NaOH})$ recorded the best tensile strength in the composite, giving 83.38 $\mathrm{MPa}$ and this condition occurs in $6 \% \mathrm{NaOH}$ at five-hour treatment. Furthermore, the highest values of elongation for treated fibres recorded was $13.21 \%$ and it occurs in $6 \% \mathrm{NaOH}$ for five-hour treatment. However, the Young modulus showed the maximum amount at $2 \%$ of $\mathrm{NaOH}$ with $2242 \mathrm{MPa}$ for one-hour treatment. From the test, sodium hydroxide treatment on pineapple leaf fibre was improved the surface structure of the prepared composite material by removing waxy materials and impurities. From the result produced by SEM, PALF that treated with $6 \% \mathrm{NaOH}$ after five-hour treatment showed the better surface structure compared to other samples.

Acknowledgements This work was supported by the Malaysian Government under Science Fund of Ministry of Science, Technology and Innovation (MOSTI) with vote No S021, Malaysian International Scholarship (MIS) under Ministry of Education Malaysia (MOE), and Universiti Tun Hussein Onn Malaysia (UTHM).

\section{References}

[1] M. Asim et al., "A Review on Pineapple Leaves Fibre and Its Composites," Int. J. Polym. Sci., vol. 2015, no. April, pp. 1-17, 2015.

[2] S. Banika,. Nag, and S. Debnath, "Utilization of pineapple leaf agro-waste for extraction of fibre and the residual biomass for vermicomposting," Indian J. Fibre Text. Res., vol. 36, no. 2, pp. 172-177, 2011.

[3] Ms. Munirah Mokhtar, Characterization and Treatments of Pineapple Leaf Fibre Thermoplastic Composite for Construction Application. 2007.

[4] A. Rijswijk, K. V., Brouwer, W.D. and Beukers, "Application of Natural Fibre Composites in the Development of Rural Societies," 2003.

[5] W. Liu, M. Misra, P. Askeland, L. T. Drzal, and A. K. Mohanty, “'Green’ composites from soy based plastic and pineapple leaf fiber: Fabrication and properties evaluation," Polymer (Guildf). vol. 46, no. 8, pp. 2710-2721, 2005.

[6] J. George, S. S. Bhagawan, and S. Thomas, "Improved interactions in chemically modified pineapple leaf fiber reinforced polyethylene composites," Compos. Interfaces, vol. 5, no. 3, pp. 201-223, 1997.

[7] K. Panyasart, N. Chaiyut, and T. Amornsakchai, "Effect of surface treatment on the properties of pineapple leaf fibers reinforced polyamide 6 composites," Energy Procedia, vol. 56, pp. 406-413, 2014.

[8] J. P. S. and M. R. M. R. 1Faculty A.A. Mohammed1, 2, D. Bachtiar1,*, "Effect of sodium hydroxide on the tensile properties of sugar palm fibre reinforced thermoplastic polyurethane composites," J. Mech. Eng. Sci., vol. 10, no. 1, pp. 17651777, 2016.

[9] Yusri. A.Anbia Adam, "Extraction of Pineapple Leaf Fibre: Josapine and Moris," vol. 1, 2016. 